LA-UR- $96=0270$

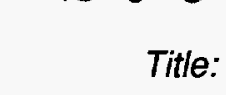

Title:

Author(s):

Submitted to:

Los Alamos

NATIONAL LABORATORY
RESULTS FROM SAGE

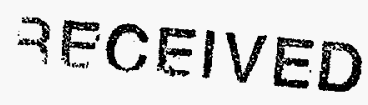

\section{APR 011996}

OS $S$

J.N. Abdurashitov, V.N. Gavrin, S.V. Girin, V.V. Gorbatchev, A.O. Gusev, A.V. Kalikhov, T.V. Knodel, I.N. Mirmov, A.M. Pshukov, A.M. Shalagin, A.A. Shikhin, E.P. Veretenkin, V.M. Vermul, V. Yants, and G.T. Zatsepin (Inst. for Nuclear Research, Russia); T.J. Bowles, J.S. Nico, W.A. Teasdale, and D.L. Wark (LANL, P-23); S.R. Elliott and J.F. Wilkerson (Univ. of Washington, Seattle); B.T. Cleveland, T. Daily, R. Davis, Jr., K.Lande, C.K. Lee, and P.W. Wildenhain (Univ. of Pennsylvania, Philadelphia); M.L. Cherry (Louisiana State Univ.)

Workshop on 'Theoretical and Phenomenological Aspects of Underground Physics' (TAUP'95), Toledo, Spain, 17-21

September 1995. Edited by M Fatas- Supplement to Nuclear Physics B.

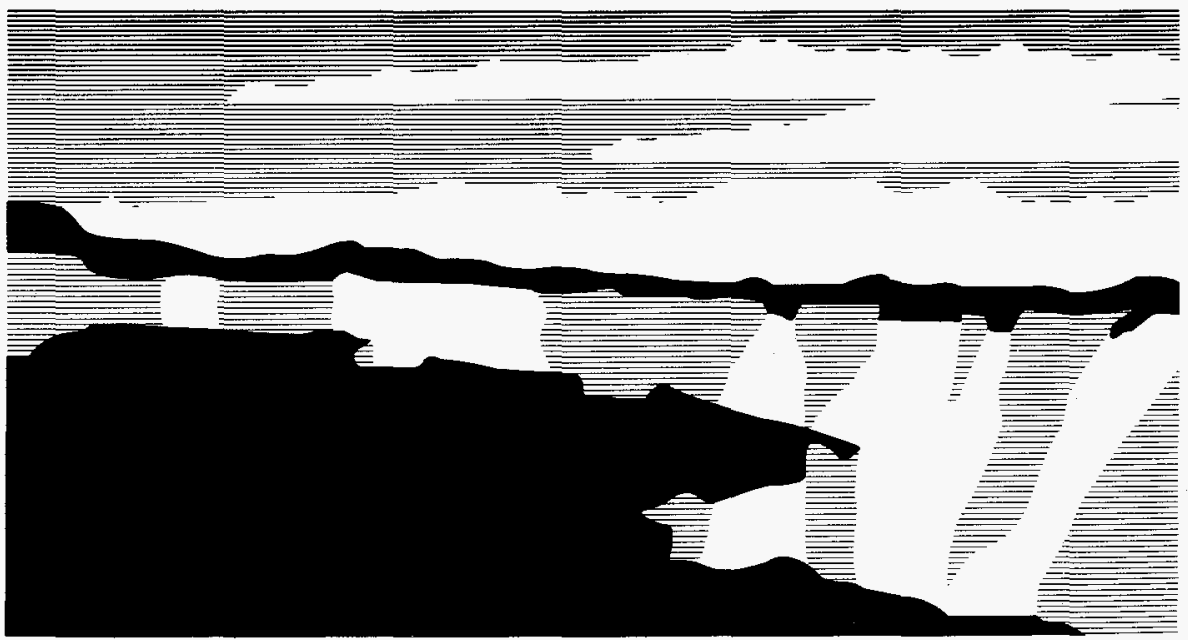

Los Alamos National Laboratory, an affirmative action/equal opportunity employer, is operated by the University of California for the U.S. Department of Energy under contract W-7405-ENG-36. By acceptance of this article, the publisher recognizes that the U.S. Government retains a nonexclusive, royalty-free license to publish or reproduce the published form of this contribution, or to allow others to do so, for U.S. Government purposes. The Los Alamos National Laboratory requests that the publisher identify this article as work pertormed under the auspices of the U.S. Department of Energy. 


\section{Results from SAGE}

J.N. Abdurashitov, V.N. Gavrin, S.V. Girin, V.V. Gorbatchev, A. O. Gusev, A.V. Kalikhov, T.V. Knodel, I.N. Mirmov, A.M. Pshukov, A. M. Shalagin, A.A. Shikhin, E.P. Veretenkin, V.M. Vermul, V. Yants, and G.T. Zatsepin

Institute for Nuclear Research, Russian Academy of Sciences, Moscow 117312, Russia

T.J. Bowles, J. S. Nico, W. A. Teasdale, and D.L. Wark*

Los Alamos National Laboratory, Los Alamos, NM 87545 USA

S.R. Elliott and J.F. Wilkerson

University of Washington, Seattle, WA 98195 USA

B.T. Cleveland, T. Daily, R. Davis, Jr., K. Lande, C.K. Lee, and P.W. Wildenhain

University of Pennsylvania, Philadelphia, PA 19104 USA

M.L. Cherry

Louisiana State University, Baton Rouge, LA 70803 USA

The Russian-American Gallium solar neutrino Experiment (SAGE) is described. Beginning in September 1992, SAGE II data were taken with 55 tons of Ga and with significantly reduced backgrounds. The solar neutrino flux measured by 31 extractions through October 1993 is presented. The result of $69 \pm 10+5 /-7$ SNU is to be compared with a Standard Solar Model prediction of 132 SNU.

\section{INTRODUCTION}

The discrepancy between the solar neutrino capture rate predicted by Standard Solar Model (SSM) calculations and the ${ }^{37} \mathrm{Ar}$ rate measured by the chlorine experiment of Davis et al. has persisted for more than twenty years [1-3]. The Kamiokande water Cerenkov experiment [4] observes only 0.50 0.64 of the ${ }^{8} \mathrm{~B}$ flux predicted by the SSMs. SAGE and GALLEX have measured rates of about $60 \%$ of the SSM predictions $[5,6]$. Thus, all four operating solar neutrino experiments have reported significant deficits of the flux of solar neutrinos.

The ${ }^{37} \mathrm{Cl}$ and Kamiokande experiments are primarily sensitive to the high-energy ${ }^{8} \mathrm{~B}$ solar neutrinos, whose production rate depends critically $\left(\propto T_{c}^{18}\right)$ on the core temperature of the Sun. Numerous nonstandard solar models $[7,8]$ that reduce the core temperature have been suggested, but none has been able to reproduce the observed ${ }^{8} \mathrm{~B}$ flux without running into difficulties accounting for other observed features of the Sun.

New particle physics $[7,8]$, such as neutrino matter oscillations, has been invoked to provide an explanation of the "solar neutrino problem." Analyses $[9,10]$ of the consistency of the chlorine and Kamiokande data conclude that the results are highly inconsistent with any astrophysical explanations and are better described by MikheyevSmirnov-Wolfenstein (MSW) neutrino oscillations. However, given the uncertainties in the SSMs, it does not seem possible to rule out an astrophysical

\footnotetext{
* Present address: Department of Particle and Nuclear Physics, Oxford University, Keble Road, Oxford, OX1 3RH, England.
} 
origin of the "solar neutrino problem" [11].

The ${ }^{71} \mathrm{Ga}\left(v_{e} e^{-}\right)^{71} \mathrm{Ge}$ reaction [12] provides the only feasible means at present to measure lowenergy solar neutrinos. The SSM calculations show that the dominant contribution to the capture rate in ${ }^{71} \mathrm{Ga}$ arises from the $p$ - $p$ neutrinos $(71 \pm 4 \mathrm{SNU})$ (1 Solar Neutrino Unit $=10^{-36}$ captures/target atom/s), while the total predicted rate in the SSMs is 122.5-131.5 SNU. [1, 2]

\section{THE BAKSAN GALLIUM EXPERIMENT}

The detector is situated in a specially built underground laboratory at the Baksan Neutrino Observatory in the Northern Caucasus Mountains. It is located $3.5 \mathrm{~km}$ from the entrance of an adit in Mount Andyrchi and has an overhead shielding of 4715 meters water equivalent. The chemical and counting procedures have been described previously and are only outlined here [5].

Each measurement of the solar neutrino flux begins by adding about $700 \mu \mathrm{g}$ of natural Ge carrier equally divided among the 8 reactors holding the 55 tonnes of Ga. After a typical 1-month exposure interval, the Ge carrier and any ${ }^{71} \mathrm{Ge}$ atoms that have been produced by neutrino capture are chemically extracted. The counting gas $\mathrm{GeH}_{4}$ (germane) is then synthesized and purified by gas chromatography. The overall extraction efficiency is typically $80 \%$.

The $\mathrm{GeH}_{4}$ is then mixed with Xe and inserted into a miniature low-background proportional counter (PC). The PC is placed in the well of a NaI veto detector inside a large passive shield and counted for 2-5 months. ${ }^{71} \mathrm{Ge}$ decays by electron capture to ${ }^{71} \mathrm{Ga}$ with an 11.4 day half life. The low-energy $K$ (10.4 keV) and $L$-shell (1.2 keV) Auger electrons and $\mathrm{X}$ rays produced during electron shell relaxation in the ${ }^{71} \mathrm{Ga}$ daughter atom are detected by the PC. Pulse-shaped discrimination based on rise time measurements is used to improve the separation of the ${ }^{71} \mathrm{Ge}$ decays from background.

The PC is typically calibrated at one- to twoweek intervals using an external ${ }^{55} \mathrm{Fe}$ source. The $K$-peak acceptance window is then determined by extrapolation from the ${ }^{55} \mathrm{Fe}$ peak. The extrapolation procedure was verified by filling a PC with ${ }^{71} \mathrm{GeH}_{4}$ together with the standard counter gas. For SAGE II, a ${ }^{109} \mathrm{Cd}-\mathrm{Se}$ fluorescence source was utilized that provides lines at $1.2,6.4$, and $11.2 \mathrm{keV}$ using the side window as well as fluorescing the entire Fe cathode to check uniformity of response.

\section{EXTRACTION HISTORY}

The experiment began operation in May of 1988 when purification of the 30 tonnes of $\mathrm{Ga}$ commenced. Solar neutrino flux extractions began in January 1990. Data from all runs made through October 1993 are reported here, except for months in which either the extraction sample was physically lost during the extraction process or runs in which no usable information was available [5] due to electronic or $\mathrm{PC}$ failures. Following completion of a ${ }^{51} \mathrm{Cr}$ engineering test run in 1990,30 tonnes of new $\mathrm{Ga}$ were purified and added to the experiment. Extractions from 57 tonnes began in September 1991. Between May 1992 and September 1992, solar neutrino runs were halted to upgrade equipment to the SAGE II configuration. With the upgrade of the counting system, the signal to background in SAGE II is substantially better than in SAGE I. The data presented here contain 31 runs through October 1993. Fourteen additional solar neutrino runs have been made since October 1993, most of which include 1-GHz transient digitization of the pulse waveform. In the SAGE II data, we are able to measure both the ${ }^{71} \mathrm{Ge} K$ and $L$ peaks.

\section{SOLAR NEUTRINO FLUX MEASUREMENTS}

\subsection{Analysis}

A standard analysis procedure for event selection was developed with two primary goals in mind: minimizing the efficiency uncertainty over the course of counting and keeping the background rate constant. 
Gain variations in the PCs were observed in some of the runs with an average variation of $3.3 \%$ (with the largest being 10\%). We accounted for these by linearly interpolating in time between ${ }^{55} \mathrm{Fe}$ calibrations bracketing the event. The frequency of calibrations was increased in later runs to minimize any uncertainty due to gain variations. In the SAGE II data, the gain shifts averaged $2.2 \%$.

After correcting for any gain variations, several cuts are made. First, a cut is made to eliminate periods of noise bursts in the data. This results in excluding $<1 \%$ of the counting time. Second, cuts are made on energy and inverse rise time that accept 2 FWHM in, and $95 \%$ of the inverse rise time distribution. Third, any event that has associated $\mathrm{NaI}$ activity is eliminated.

Fourth, a cut was made to eliminate possible backgrounds from $\mathrm{Rn}$ daughters on the external surfaces of the PCs. Removing all data within 1.0 hour of a shield opening eliminates any observable excess of $\mathrm{Rn}$ in the $K$-peak acceptance window.

A maximum likelihood analysis [14] is then carried out by fitting the time distribution to a ${ }^{71} \mathrm{Ge}$ exponential decay plus a constant rate background. The ${ }^{71} \mathrm{Ge}$ half life is fixed at 11.43 days and unphysical regions of parameter space are excluded.

The systematic uncertainties determined for the full SAGE data set have been described in detail elsewhere [5] and are only summarized in Table 1.

Table 1

Systematic uncertainties $(1-\sigma)$ for the combined 1990-93 data sets.

\begin{tabular}{lr} 
Chemical Extraction Efficiency & \pm 2.5 SNU \\
Counting Efficiency & $+2.9 /-2.1$ SNU \\
K-peak Acceptance & $+3.5 /-0.7$ SNU \\
Backgrounds & -1.3 SNU \\
Radon & -5.9 SNU \\
TOTAL & $+5.2 /-6.9$ SNU \\
\hline
\end{tabular}

Figure 1 shows the individual run results and the combined result. The 31 solar neutrinos runs were compared with the predicted from 1000 Monte Carlo simulations of each extraction using the combined fit parameters and the constants of each data set. The two distributions are quite similar and the data are distributed according to Poisson statistics. The halflife for the decay measured from the combined data sets is $12 \pm 3$ days, in good agreement with the known ${ }^{71} \mathrm{Ge}$ half-life of 11.4 days.

\subsection{Extraction efficiencies}

In order to test the assumption that the extraction efficiency for ${ }^{71} \mathrm{Ge}$ atoms produced by solar neutrinos is the same as for the carrier, several tests have been carried out. First, Ge carrier doped with a known number of ${ }^{71} \mathrm{Ge}$ atoms was added to a reactor holding 7 tonnes of $\mathrm{Ga}$. Three successive extractions were carried out, and the number of ${ }^{71} \mathrm{Ge}$ atoms in each extraction was determined by counting. The chemical extraction efficiency was $101 \pm 5 \%$ and the ${ }^{71} \mathrm{Ge}$ extraction efficiency was 99 $+6 /-8 \%$. Second, measurements was made by observing the beta decay of radioactive $\mathrm{Ga}$ isotopes in liquid $\mathrm{Ga}$. The extraction efficiencies were $98 \pm$ $10 \%(92 \pm 10 \%)$ for ${ }^{70} \mathrm{Ge}\left({ }^{72} \mathrm{Ge}\right)$ produced by the beta decay of ${ }^{70} \mathrm{Ga}\left({ }^{72} \mathrm{Ga}\right.$.

Finally, an experiment using a ${ }^{51} \mathrm{Cr}$ neutrino source to test the overall extraction efficiency in situ is reported elsewhere in these proceedings.

\subsection{Results}

The best fit value for the capture rate and the uncertainties for the combined 1990-93 solar neutrino data are:

$$
{ }^{71} \mathrm{Ga} \text { Rate }=69 \pm 10 \text { (stat) }+5 /-7 \text { (syst) SNU. }
$$

This assumes that the extraction efficiency for ${ }^{71} \mathrm{Ge}$ atoms produced by solar neutrinos is the same as that measured using natural $\mathrm{Ge}$ carrier. 


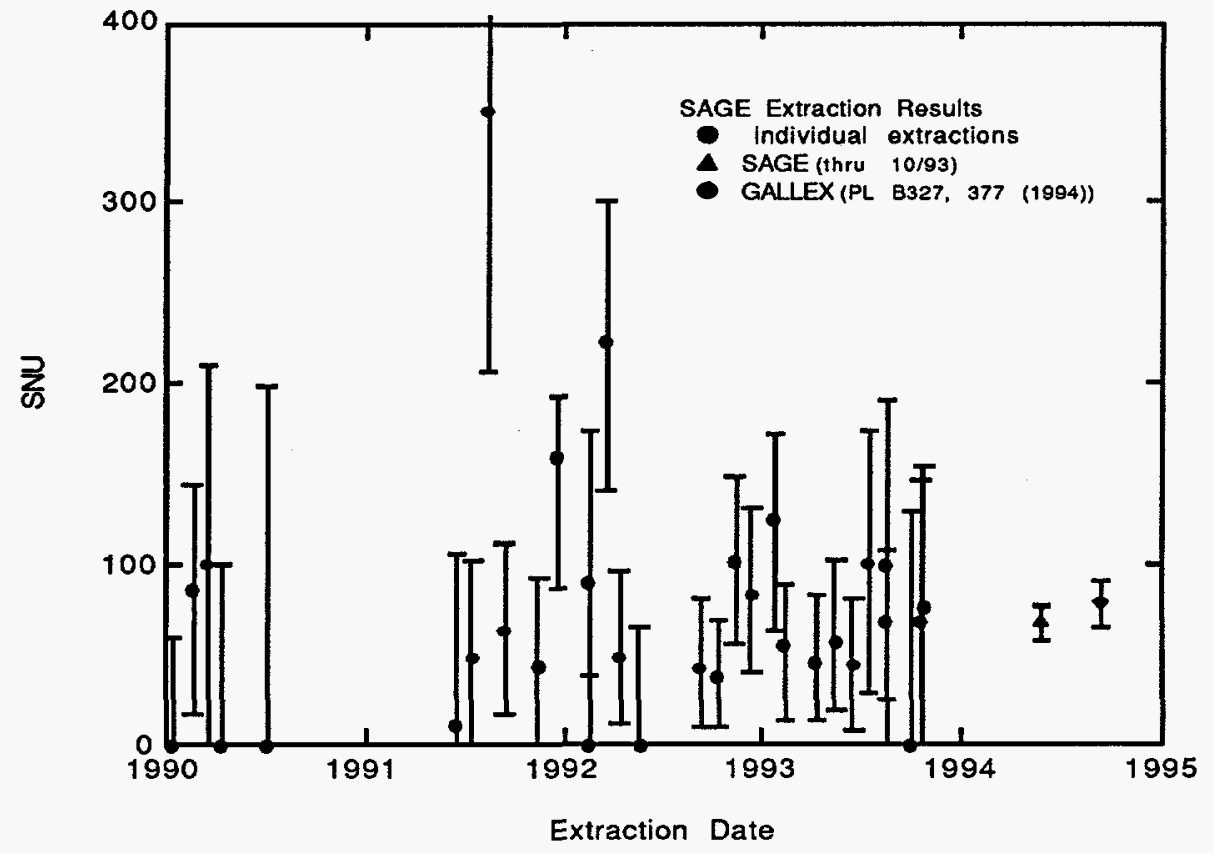

Figure 1. Best fit values and 1- $\sigma$ uncertainties for each of the runs, together with the best fit value and 1- $\sigma$ uncertainty for the combined $1990-93$ data.

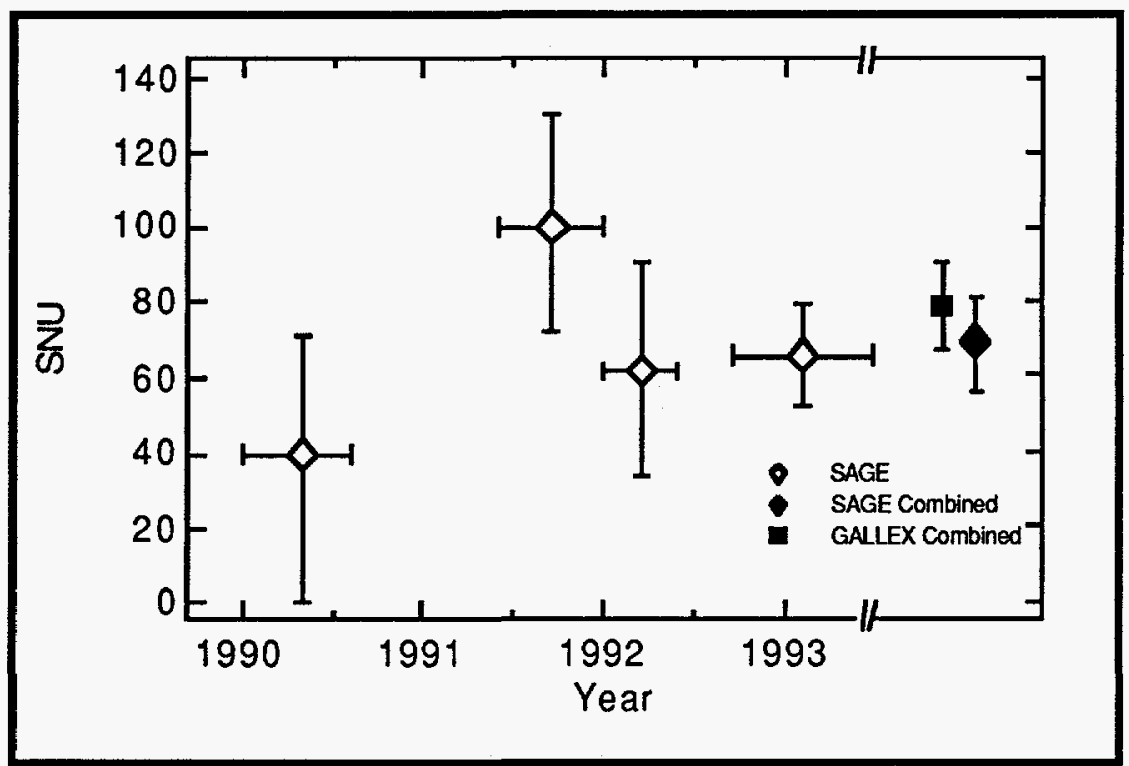

Figure 2. Results from SAGE (diamond) for measurements from 1990-1993. Also shown are the current SAGE and GALLEX [6] results from all runs reported. The errors shown are derived by adding the quoted statistical and systematic uncertainties in quadrature. The two values shown for the SAGE 1990 data are from the original (light line) and new analyses (diamond). 


\section{CONCLUSIONS}

The measurements made by SAGE from January 1990 through October 1993 have observed fewer ${ }^{71} \mathrm{Ge}$ atoms than predicted by the SSMs. From the 1990-93 data, SAGE observes only $56 \%$ to $60 \%$ of the predicted Bahcall-Pinsonneault and Turck-Chieze rates, respectively. $[1,2]$

Taken alone, the SAGE result appears to favor a non-astrophysical solution of the "solar neutrino problem," but cannot rule out an astrophysical solution. Taken together with the other operating solar neutrino experiments, it seems that astrophysical solutions are improbable, as discussed by a number of authors [9-10,15-16].

The solar neutrino experiments are consistent with two possible MSW solutions. The "nonadiabatic" solution (with $\Delta m^{2} \approx 6 \times 10^{-6} \mathrm{eV}^{2}$ and $\sin ^{2} 2 \theta \approx 7 \times 10^{-3}$ ) is the favored solution and represents a strong suppression of ${ }^{7} \mathrm{Be}$ neutrinos, a significant suppression of ${ }^{8} \mathrm{~B}$ neutrinos, and essentially no suppression for the $p$ - $p$ neutrinos. Alternately, for the large-mixing angle solution (with $\Delta m^{2} \approx 10^{-5} \mathrm{eV}^{2}$ and $\sin ^{2} 2 \theta \approx 0.8$ ), it may be that all solar neutrinos are suppressed approximately the same, roughly independent of energy.

With the improved precision from the combined 1990-93 SAGE data, SAGE is seeing approximately the rate predicted for the $p-p$ neutrinos alone. With the ability to count both the $K$ and $L$ peaks with 55 tonnes of gallium, SAGE expects to reach a precision within the next two years that should provide a strong test for astrophysical solutions to the "solar neutrino problem."

\section{ACKNOWLEDGMENTS}

The SAGE collaboration wishes to thank J.N. Bahcall, A.E. Chudakov, V.A. Matveev, and V.A. Rubakov, A. Yu. Smirnov, and many members of the GALLEX collaboration for their continued interest in our work and for stimulating discussions. We acknowledge the support in Russia of the Russian Academy of Sciences, the Institute for Nuclear Research, the Russian Ministry of Science and Technology, the Russian Foundation of
Fundamental Research, the Department of Energy, the National Science Foundation, and Los Alamos National Laboratory. The research described in this publication was made possible in part by Grant M7F000 from the International Science Foundation and Grant M7F300 from the International Science Foundation and the Russian Government.

\section{REFERENCES}

1. J.N. Bahcall and M.H. Pinsonneault, Rev. Mod. Phys. 64 (1992) 885.

2. S. Turck-Chieze and I. Lopes, Astrophys.. Jour. 408 (1993) 347.

3. R. Davis, Prog. Part. Nucl. Phys., 32 (1994), in press.

4. K.S. Hirata et al., Phys. Rev. Lett. 65 (1990) 1297, Y. Totsuka, Proc. Texas/PASCOS 92: Relativistic Astrophysics and Particle Cosmology, Annals of the New York Academy of Sciences, Vol. 688 (1993) 344.

5. J.N. Abdurashitov et al., (SAGE Collaboration), Phys. Lett. 328B (1994) 234.

6. P. Anselmann et al., (GALLEX Collaboration), to appear in Phys. Lett. B (1994).

7. J.N. Bahcall, Neutrino Astrophysics (Cambridge Univ. Press, 1989).

8. T.J. Bowles and V.N. Gavrin, Annu. Rev. Nucl. Part. Sci. 43 (1993) 117.

9. H. A. Bethe and J. N. Bahcall, Phys. Rev. D44 (1991) 2962.

10. S.A. Bludman, N. Hata, D.C. Kennedy, and P.G. Langacker, Phys. Rev. D47 (1993) 2220.

11. S. Turck-Chieze, Nucl. Phys. B31 (1993) 129.

12. V.A. Kuzmin, Zh. Eksp. Teor. Fiz. 49 (1965) 1532, Sov. Phys. JETP 22 (1966) 1051.

13. A.I. Abazov et al., Phys. Rev. Lett. 67 (1991) 3332.

14. B.T. Cleveland, Nucl. Instrum. Methods 214 (1983) 451.

15. L. Krauss, E. Gates, and M. White, Phys. Lett. 299B (1993) 94.

16. P.I. Krastev and S.T. Petcov, Phys. Lett. 299B (1993) 99. 


\section{DISCLAIMER}

This report was prepared as an account of work sponsored by an agency of the United States Government. Neither the United States Government nor any agency thereof, nor any of their employees, makes any warranty, express or implied, or assumes any legal liability or responsibility for the accuracy, completeness, or usefulness of any information, apparatus, product, or process disclosed, or represents that its use would not infringe privately owned rights. Reference herein to any specific commercial product, process, or service by trade name, trademark, manufac: turer, or otherwise does not necessarily constitute or imply its endorsement, recommendation, or favoring by the United States Government or any agency thereof. The views and opinions of authors expressed herein do not necessarily state or reflect those of the United States Government or any agency thereof. 